\title{
Fatores de risco para hipertensão arterial sistêmica em idosas de um centro de referência
}

\author{
Risk factors for systemic hypertension in elderly women in a reference center
}

Tainá Cristina Lara Assunçãoa, Graziela De Carlib, Ana Luisa Sant'Anna Alvesc, Daniela Bertold, Juliane Berviane, Marlene Doringf, Marilene Rodrigues Portellag, Helenice de Moura Scortegagnag, Júlia Pancotteh ${ }^{h}$ Emanuelly Casal Bortoluzzii, Bernadete Maria Dalmolini

Nutricionista graduada Universidade de Passo Fundo (UPF), RS, Brasil.

b Nutricionista. Mestre Ciência e Tecnologia de Alimentos pela UPF. Docente do Curso de Nutrição da UPF.

c Nutricionista. Doutora em Epidemiologia pela Universidade Federal do Rio Grande do Sul (UFRGS). Docente do Curso de Nutrição da UPF.

d Fisioterapeuta. Mestre em Epidemiologia pela UFRGS. Coordenadora do Centro de Referência e Atenção ao Idoso - CREATI e docente no Programa de Fisioterapeuta. Mestre em Epidemiologia pela UFRGS. Coordenadora do Ce
Residência Multiprofissional Integrada e Faculdade de Medicina da UPF.

e Dentista. Doutora em Odontologia pela Universidade Luterana do Brasil (ULBRA). Docente do Curso de Odontologia da UPF.

Enfermeira. Doutora em Saúde Pública pela Universidade de São Paulo (USP). Docente do Instituto de Ciências Biológicas e do Programa de Mestrado em Envelhecimento Humano da UPF.

g Enfermeira. Doutora em Enfermagem pela Universidade Federal de Santa Catarina (UFSC). Docente do Instituto de Ciências Biológicas e do Programa de Mestrado em Envelhecimento Humano da UPF.

h Fisioterapeuta. Mestre em Envelhecimento Humano pela UPF.

Educadora física. Mestre em Envelhecimento Humano pela UPF. Docente do Instituto de Desenvolvimento Educacional do Alto Uruguai, Getúlio Vargas, RS.

Enfermeira. Doutora em Saúde Pública pela USP. Docente e pesquisadora da UPF.

RESUMO Introdução: Há evidência de que conforme os indivíduos envelhecem, as doenças crônicas não transmissíveis tornam-se as principais causas de morbidade, incapacidades e mortalidade em todo o mundo.

Objetivo: Este trabalho buscou identificar fatores de risco para hipertensão arterial sistêmica em idosas de um Centro de Referência e Atenção ao Idoso.

Materiais e Métodos: Este estudo transversal está aninhado no Estudo Longitudinal do Centro de Referência e Atenção ao Idoso (Elo-Creati). Os fatores de risco investigados foram: consumo de bebidas alcóolicas, uso de tabaco, história familiar de hipertensão arterial sistêmica, nível de atividade física e estado nutricional. Também foram investigadas variáveis demográficas e socioeconômicas. A análise dos dados foi realizada por meio de frequências absoluta e relativa simples e teste Qui-quadrado.

Resultados: Foram avaliadas 313 idosas, sendo 262 (84,5\%) de cor branca, a maioria com idade entre 60 a $69(57,2 \% ; n=179)$ anos e pertencente a classe econômica B $(52,1 \% ; n=162)$. Das idosas participantes deste estudo, $187(59,9 \%)$ referiram a presença de hipertensão arterial sistêmica, e os fatores associados encontrados foram: classe econômica, IMC e nível de atividade física $(p<0,05)$.

Conclusão: Entre os fatores de risco estudados, destaca-se a presença de história familiar de hipertensão arterial sistêmica, excesso de peso e inatividade física. Assim, sugere-se ações dos órgãos públicos e setores privados no enfrentamento efetivo desta realidade.

Palavras-chave: estado nutricional; envelhecimento; doença crônica.

ABSTRACT

Introduction: There is evidence that as individuals age, chronic non-communicable diseases become the leading causes of morbidity, disability, and mortality worldwide.

Objective: This study aimed to identify risk factors for systemic arterial hypertension in the elderly of a Center for Reference and Care for the Elderly.

Materials and Methods: This cross-sectional study is nestled in the Longitudinal Study of the Center for Reference and Attention to the Elderly (Elo-Creati). The risk factors investigated were: alcohol consumption, tobacco use, family history of systemic arterial hypertension, level of physical activity and nutritional status. Demographic and socioeconomic variables were also investigated. Data analysis was performed using absolute and relative simple frequencies, and Chi-square test.

Results: A total of 313 elderly women were evaluated, 262 (84.5\%) were white, the majority ranging from 60 to 69 years old (57.2\%; $n=179)$ and belonging to economic class B $(52.1 \% ; n=162)$. From all female elderly participants in this study, $187(59.9 \%)$ reported systemic arterial hypertension, and the associated factors were: economic class, BMI and level of physical activity $(p<0.05)$.

Conclusion: Among the risk factors studied, family history of hypertension, overweight and physical inactivity were more prevalent. Thus, actions of public agencies and private sectors are suggested to effective face this reality.

Keywords: nutritional status; aging; chronic disease. 


\section{INTRODUÇÃO}

O Brasil, em 2025, será o sexto país do mundo em número de idosos ${ }^{1}$. Dentre os fatores que contribuem para este aumento, destaca-se a redução da taxa de natalidade e mortalidade e o progresso do conhecimento sobre a prevenção e o tratamento das doenças².

Apesar deste acréscimo na expectativa de vida e no avanço da medicina, é crescente a prevalência de fatores de risco para as doenças crônicas não transmissíveis (DCNT) ${ }^{3}$. Há evidência que conforme os indivíduos envelhecem, as DCNT tornam-se as principais causas de morbidade, incapacidades e mortalidade em todo o mundoํ․ Nesse sentido, há necessidade de vigilância dos principais fatores de risco, como tabagismo, consumo excessivo de álcool, inatividade física, excesso de peso, consumo inadequado de frutas e hortaliças e hiperglicemia ${ }^{3}$. Desta forma, o diagnóstico precoce das DCNT e a identificação dos fatores de risco são determinantes para evitar o crescimento epidêmico dessas doenças e seus efeitos na qualidade de vida e no sistema de saúde ${ }^{4}$.

A Hipertensão Arterial Sistêmica (HAS) é uma doença de grande prevalência na população e a sua multiplicidade de consequências e o impacto dos efeitos colaterais dos medicamentos, caracterizam-se como uma das causas de maior redução de qualidade e expectativa de vida dos indivíduos ${ }^{4}$.

No Brasil, 30\% das pessoas adultas são hipertensas, chegando a mais de $50 \%$ na terceira idade e $5 \%$ em crianças e adolescentes ${ }^{5}$, sendo que alguns dos fatores de risco importantes são passíveis de serem alterados em busca de uma alimentação saudável, de atividades físicas rotineiras e do abandono de substâncias como álcool e o tabaco. Estudos mostram que estas práticas são melhor vivenciadas e sistematizadas em grupos de convivência. Os idosos que pertencem a estes grupos referem diferença na capacidade funcional, no estado geral de saúde, na vitalidade e, sobre tudo, grande melhora nos aspectos sociais ${ }^{6-8}$.

Considerando esse contexto, o objetivo deste trabalho foi identificar alguns fatores de risco para hipertensão arterial sistêmica em idosas de um Centro de Referência e Atenção ao Idoso (Creati) do município de Passo Fundo, RS. O Creati é um local em que os idosos realizam atividades educacionais, físicas, técnica, mentais, culturais, sociais, cívicas e afetivas pensando em preservar a qualidade de vida na longevidade, assim estudos realizados com essa população servem de subsídio para ações de educação e promoção da saúde na velhice.

\section{MATERIAIS E MÉTODOS}

Estudo transversal aninhado ao estudo longitudinal do Centro de Referência e Atenção ao Idoso (Elo-creati). O projeto foi aprovado pelo Comitê de Ética em Pesquisa da Universidade de Passo Fundo (parecer no 741.214). Foram convidadas a participar do estudo todas as idosas matriculadas em oficinas oferecidas pelo Centro de Referência e Atenção ao Idoso (Creati). Desta forma, o processo de amostragem foi por conveniência. Por meio do Termo de Consentimento Livre e Esclarecido preservou-se a identidade das idosas que aceitaram participar. Os dados foram coletados de setembro de 2014 a agosto de 2015, sendo utilizados neste estudo somente os dados dos participantes do sexo feminino.

Para o cálculo da amostra, levou-se em consideração uma população de 400 idosas, prevalência da doença de $50 \%$, erro aceitável de $5 \%$ e nível de confiança de $95 \%$, totalizando 196 idosas, no entanto, optou-se em incluir no estudo todas as idosas.

Para a avaliação da presença de HAS foi utilizado o relato de diagnóstico prévio de HAS. Destaca-se que as idosas presentes na amostra são ativas, alfabetizadas e com bom nível socioeconômico. Estudos nacionais como, o Sistema de Vigilância de Fatores de Risco e Proteção para DCNT (VIGITEL), também utilizam o autorrelato de HAS para descrever a prevalência da doença na população brasileira.

Foram coletados dados socioeconômicos, demográficos, fatores de risco para HAS e aferidas medidas antropométricas. Para avaliar as condições socioeconômicas, seguiu-se o Critério de Classificação Econômica Brasil da ABEP (Associação Brasileira de Empresas de Pesquisa) ${ }^{9}$. As variáveis demográficas coletadas foram idade, estado civil e cor da pele. Questionou-se o uso de bebidas alcóolicas (sim ou não), uso de tabaco (não fumante, fumante ou ex-fumante) e história familiar (sem histórico familiar, se histórico de pai e mãe com HAS, somente mãe, somente pai).

Para avaliação do nível de atividade física foi aplicado o International Physical Activity Questionnaire (IPAQ), versão curta. Os níveis de atividade física foram calculados com base nos Guidelines for Data Processing and Analysis of the $I P A Q^{10}$, a partir das informações de dias de atividade física utilizando o valor de Metabolic Equivalent Task (MET) correspondente para atividade.

Para a avaliação do estado nutricional, aferiram-se as medidas de peso e altura em triplicata e classificado com os parâmetros de referência ${ }^{11}$. O peso foi aferido através de balança digital da marca Plenna ${ }^{\circledR}$ e para a estatura utilizou-se o estadiômetro da marca Seca ${ }^{\circledR}$. O IMC foi classificado em baixo peso $\left(<23 \mathrm{~kg} / \mathrm{m}^{2}\right)$, eutrófico $\left(\geq 23 \mathrm{e}<28 \mathrm{~kg} / \mathrm{m}^{2}\right)$ e excesso de peso (sobrepeso e obesidade) $\left(\geq 28 \mathrm{~kg} / \mathrm{m}^{2}\right)^{11}$.

Os dados foram digitados e analisados em software de estatística. Para as variáveis qualitativas foram apresentadas as frequências absoluta e relativa simples. A associação 
entre HAS e os fatores de risco foi testada por meio do teste qui-quadrado, nível de significância de 5\%.

\section{RESULTADOS}

Foram investigadas 313 idosas, a maioria de cor branca $(84,5 \% ; n=262)$, entre 60 a 69 anos $(57,2 \% ; n=179)$, não possuíam companheiro $(62,3 \% ; n=195)$ e pertencia a classe econômica B (52,1\%; $n=162)$ (Tabela 1).

A prevalência de HAS encontrada na amostra estudada foi de $59,9 \%(n=187)$. Quanto aos fatores de risco investigados, verificou-se que cerca de 57,9\% $(n=172)$ das idosas possuíam história familiar de doença hipertensiva, 21,7\% $(n=68)$ eram ex-fumantes, $12,8 \%(n=40)$ das entrevistadas

Tabela 1. Características demográficas e socioeconômicas das idosas de um Centro de Referência e Atenção ao Idoso $(n=313)$.

\begin{tabular}{llcc}
\hline Variáveis & Categorias & $\mathbf{n}$ & $\mathbf{\%}$ \\
Idade & 60 a 69 anos & 179 & 57,2 \\
& 70 a 79 anos & 113 & 36,1 \\
\multirow{2}{*}{ Estado civil } & 80 a 89 anos & 21 & 6,7 \\
& Divorciada/Separada & 37 & 11,8 \\
& Viúva & 117 & 37,4 \\
& Casada/União estável & 118 & 37,7 \\
Cor da pele & Solteira & 41 & 13,1 \\
& Branca & 262 & 84,5 \\
Classe econômica & Não-branca & 48 & 15,4 \\
& Classe A & 17 & 5,5 \\
& Classe B & 162 & 52,1 \\
& Classe C & 122 & 39,2 \\
& Classe D & 10 & 3,2 \\
\hline
\end{tabular}

ingeriam bebidas alcóolicas, 47\% $(n=147)$ das idosas eram suficientemente ativas e $31,5 \%(n=92)$ apresentaram algum grau de excesso de peso (Tabela 2 ).

A Tabela 3 apresenta a associação entre HAS autorreferida e os fatores de risco. As variáveis classe econômica e índice de massa corporal tiveram associação significativa com a presença de HAS ( $p=0,004$ e $p \leq 0,001$, respectivamente), sendo que a prevalência de HAS foi maior entre as idosas pertencentes às classes econômicas C e D $(68,9 \% ; n=91)$ e com excesso de peso $(78,3 \% ; n=72)$.

Tabela 2. Descrição das variáveis de risco para hipertensão arterial sistêmica das idosas de um Centro de Referência e Atenção ao Idoso $(n=313)$.

\begin{tabular}{llcc}
\hline Variáveis & Categorias & $\mathbf{n}$ & $\mathbf{\%}$ \\
IMC & Baixo peso & 46 & 15,8 \\
& Eutrófico & 154 & 52,7 \\
Nível de atividade física & Excesso de peso & 92 & 31,5 \\
& Insuficientemente ativo & 132 & 42,2 \\
& Suficientemente ativo & 147 & 47,0 \\
História de HAS & Muito ativo & 34 & 10,9 \\
& Não & 125 & 42,1 \\
& Pai e mãe & 48 & 16,2 \\
Uso de tabaco & Somente mãe & 91 & 30,6 \\
& Somente pai & 33 & 11,1 \\
& Não fumante & 235 & 75,1 \\
Bebida alcoólica & Fumante & 10 & 3,2 \\
& Ex-fumante & 68 & 21,7 \\
& Sim & 40 & 12,8 \\
& Não & 273 & 87,2 \\
\hline
\end{tabular}

IMC: índice de massa corporal; HAS: hipertensão arterial sistêmica.

Tabela 3. Associação entre hipertensão arterial sistêmica e fatores de riscos das idosas de um Centro de Referência e Atenção ao Idoso ( $n=313$ ).

\begin{tabular}{|c|c|c|c|c|c|c|}
\hline \multirow{3}{*}{ Variáveis } & \multirow{3}{*}{ Categorias } & \multicolumn{4}{|c|}{ Hipertensão } & \multirow{3}{*}{ valor de $p^{*}$} \\
\hline & & \multicolumn{2}{|c|}{ Sim } & \multicolumn{2}{|c|}{ Não } & \\
\hline & & $\mathbf{n}$ & $\%$ & $\mathbf{n}$ & $\%$ & \\
\hline \multirow[t]{3}{*}{ Idade } & 60 a 69 anos & 97 & 54,2 & 82 & 45,8 & 0,054 \\
\hline & 70 a 79 anos & 77 & 68,1 & 36 & 31,9 & \\
\hline & 80 a 89 anos & 13 & 65,0 & 7 & 35,0 & \\
\hline \multirow[t]{2}{*}{ Estado civil } & Com companheiro & 124 & 63,6 & 71 & 36,4 & 0,057 \\
\hline & Sem companheiro & 63 & 53,8 & 54 & 46,2 & \\
\hline \multirow[t]{2}{*}{ Cor da pele } & Branco & 155 & 59,4 & 106 & 40,6 & 0,406 \\
\hline & Não Branco & 30 & 62,5 & 18 & 37,5 & \\
\hline \multirow[t]{2}{*}{ Classe econômica } & Classe A e B & 95 & 53,4 & 83 & 46,6 & 0,004 \\
\hline & Classe C e D & 91 & 68,9 & 41 & 31,1 & \\
\hline \multirow[t]{3}{*}{ Índice de massa corporal } & Baixo peso & 20 & 43,5 & 26 & 56,5 & $<0,001$ \\
\hline & Eutrófico & 83 & 54,2 & 70 & 45,8 & \\
\hline & Excesso de peso & 72 & 78,3 & 20 & 21,7 & \\
\hline \multirow[t]{3}{*}{ Nível de atividade física } & Insuficientemente ativo & 79 & 59,8 & 53 & 40,2 & 0,045 \\
\hline & Suficientemente ativo & 94 & 64,4 & 52 & 35,6 & \\
\hline & Muito ativo & 14 & 41,2 & 20 & 58,8 & \\
\hline \multirow[t]{3}{*}{ Uso de tabaco } & Fumante & 4 & 40,0 & 6 & 60,0 & 0,171 \\
\hline & Não fumante & 137 & 58,5 & 97 & 41,5 & \\
\hline & Ex-fumante & 46 & 67,6 & 22 & 32,4 & \\
\hline \multirow[t]{2}{*}{ Bebida alcoólica } & $\operatorname{Sim}$ & 21 & 52,5 & 19 & 47,5 & 0,196 \\
\hline & Não & 166 & 61,0 & 106 & 39,0 & \\
\hline
\end{tabular}

* Significância estatística com $p<0,05$. 
As demais variáveis não apresentaram associação significativa, porém percebeu-se maior prevalência de HAS em idosas com maior idade $(68,1 \% ; p=0,054)$, que viviam com companheiro $(63,6 \% ; p=0,057)$, com cor de pele não branca $(62,5 \% ; p=0,406)$, ex-fumantes $(67,6 \%$; $p=0,171)$ e que não faziam uso de bebida alcoólica $(61 \%$; $p=0,196)$.

E, em relação ao nível de atividade física, encontramos uma menor prevalência, estatisticamente significativa, de HAS em idosas muito ativas comparados com níveis baixos de atividade física $(41,2 \% ; p=0,045)$.

\section{DISCUSSÃO}

Este estudo mostrou a alta prevalência tanto da hipertensão arterial (59,9\%), quanto dos fatores associados a ela: classe econômica, IMC e nível de atividade física. No que se refere à prevalência e fatores associados, a Pesquisa Nacional de Saúde realizada em 2013, identificou que a prevalência de hipertensão foi de $21,4 \%$, sendo maior em mulheres $(24,2 \%)$, em indivíduos com mais de 75 anos (55\%), com menor escolaridade $(31,1 \%)$, de cor preta $(24,2 \%)$, residentes em zona urbana (21,7\%), no Rio Grande do Sul $(24,9 \%)$ e na região Sudeste $(23,3 \%)^{12}$. Destaca-se que, este estudo em Passo Fundo, possui uma similaridade nos resultados das prevalências dos fatores de risco, sendo relevantes estudos no Rio Grande do Sul para avaliar características próprias de amostras menores e regionais.

No presente estudo, verificou-se que a maioria das mulheres idosas apresenta hipertensão (59,9\%; $n=187)$, resultado superior foi observado no estudo realizado em Goiânia, em que $82,6 \%$ das idosas eram hipertensas ${ }^{15}$. A amostra de idosas de Goiânia foi composta por usuárias do SUS e em nível domiciliar, já a amostra do presente estudo, por idosas que frequentam um centro de referência, isso pode explicar, em parte, a menor prevalência de hipertensão.

Estudo realizado com idosos não institucionalizados também observou elevada prevalência de HAS (82,3\%) e ressalta os resultados que evidenciam as idosas como mais vulneráveis a ocorrência de doenças cardiovasculares por apresentarem maior prevalência dos fatores de risco ${ }^{16}$. Estudo realizado por Zattar et al. ${ }^{17}$ também identificou elevada prevalência de HAS $(84,6 \%)$. Os autores referem a desigualdade na distribuição da doença na população e a necessidade de ações de prevenção, diagnóstico e garantia de tratamento.

A experiência de outros países mostra que o sucesso das intervenções de saúde pública no que se refere aos fatores de risco e à redução da prevalência das doenças e agravos não transmissíveis tem sido atribuído ao enfoque na vigilância de fatores comuns de risco e na promoção de modos de viver favoráveis à saúde e à qualidade de vida, tendo um custo menor do que as abordagens para as doenças específicas ${ }^{1,3,18}$. Atitudes negligentes com aspectos preventivos, propiciam um somatório de outros agravos com o aumento da idade, diminuindo com isso, a possibilidade de controle da doença e aumento excessivo do custo da manutenção de vida desta pessoa.

No entanto, estudo realizado no município de Bambuí (MG) identificou que $26,9 \%$ das idosas apresentavam hipertensão. E, embora esta prevalência seja baixa em relação à maioria dos estudos, os autores também observaram que a hipertensão foi associada à inatividade física, ao excesso de peso, a hiperglicemia, hipercolesterolemia e a hipertrigliceridemia ${ }^{19}$. Apesar da diferença entre as prevalências, na amostra de idosas de Passo Fundo, também foi observada maior prevalência de HAS nas idosas com excesso de peso e com menor nível de atividade física.

Em relação à classe econômica, os resultados do presente estudo demonstram maior prevalência de hipertensão arterial nas classes econômicas C e D $(68,9 \% ; n=91)$. No mesmo sentido, estudo realizado na cidade de Catanduva (SP), investigou indivíduos acima de 18 anos de idade $(n=688)$ e identificou que 31,5\% apresentavam hipertensão e a doença esteve associada aos indivíduos com renda familiar de 1 a 5 salários mínimos $(29,79 \%)^{20}$. Ambos estudos evidenciam a tendência da associação entre HAS e pessoas de baixa renda e/ou de classe econômica menos favorecida.

Quanto ao estado nutricional, o presente estudo evidenciou a associação entre o excesso de peso e a HAS. No estudo de Bambuí citado anteriormente, os idosos com sobrepeso e obesidade apresentaram maior suscetibilidade de HAS comparado com os idosos eutróficos. Neste estudo, os autores incentivam novos estudos epidemiológicos regionais com desfecho e fatores associados similares, indicando que cerca de 27 milhões de brasileiros vivem em pequenas cidades, como o investigado neste estudo, e muito pouco se sabe sobre suas condições de saúde. Porque não há motivos para supor que a população de Bambuí difere daquelas de outras cidades de tamanho similar, é razoável investigar sobre a extensão e as implicações para os serviços de saúde do perfil de risco cardiovascular retratado neste estudo ${ }^{19}$.

No que diz respeito ao nível de atividade física, as idosas insuficientemente ativas, de nosso estudo, apresentaram maior prevalência de HAS e as classificadas como muito ativas, menor prevalência de HAS (respectivamente 59,8\% e $41,2 \% ; p=0,045)$. Estudo experimental realizado no Hospital de Clínicas da Universidade Federal de Goiás apresentou esta relação com idosos diagnosticados com nível I de HAS e sem uso de medicamentos. O grupo experimental foi submetido 
a atividade física supervisionada. Após 6 meses de estudo, os autores concluíram que a atividade física foi eficiente em manter o controle da pressão arterial ${ }^{21}$.

A prática de atividade física orientada apresenta-se eficiente na execução do tratamento não-farmacológico da hipertensão, exercícios aeróbicos associados com musculação, também determinantes, demonstrando assim, a importância no incentivo de atividade física para o idoso ${ }^{21}$. Os resultados aqui mencionados podem estar relacionados ao fato das idosas sentirem-se ativas por estar em um Centro de referência, mas trata-se de um estudo transversal e os dados não correspondem a atividade física supervisionada e acompanhada por estudos longitudinais.

A redução da prevalência de HAS influencia diretamente na redução da mortalidade prematura $(<70$ anos) por DCNT $^{22}$. Portanto, a identificação de fatores de risco para HAS em grupos específicos possibilita a ações de prevenção e tratamento precoce. Desta forma, sugere-se ações dos órgãos públicos e setores privados no enfrentamento desta realidade ${ }^{18}$. Assim como, o monitoramento e avaliação das ações realizadas, uma vez que, a redução da exposição dos idosos aos fatores de risco investigados pode melhorar a expectativa e qualidade de vida deste grupo populacional.

As doenças cardiovasculares têm sido alvo de inúmeros estudos epidemiológicos, o que possibilitou identificar diversos fatores de risco, entre eles a hipertensão arterial sistêmica, motivo deste nosso estudo. Nesses inquéritos, a prevalência de hipertensão arterial tem sido obtida através da aferição da pressão arterial ou do auto-relato ${ }^{12,13}$. Embora a aferição da pressão seja uma forma de alcançar maior acurácia, implica maiores custos e maior complexidade na logística da coleta de dados, uma vez que, além de envolver o uso de equipamentos, poderá exigir a revisita aos participantes, pois reflete uma avaliação pontual ${ }^{14}$. Por outro lado, a aplicação de questionários, buscando a informação autorreferida, é uma estratégia de menor custo, acessível e rápida para estimar a prevalência de hipertensão arterial. Esta metodologia, justificada pela literatura ${ }^{12-14}$ e aplicada neste estudo, possui como condição impar o fato da amostra conter idosas independentes, não institucionalizadas, alfabetizadas e, que frequentam sozinhas um grupo de convivência.

Levando em conta os aspectos diferenciados e a propriedade deste estudo em relacionar a Hipertensão à fatores associados importantes, cabe, com a mesma clareza mencionar limitações do estudo a serem observadas. As interpretações e inferências devem ser realizadas com cautela, pois, trata-se de um estudo transversal com amostra de um grupo de idosas, em que existe possibilidade de viés de memória e causalidade reversa. Além do viés de diagnóstico, uma vez que, foi autorrelato, não foi verificada a pressão arterial e a utilização de medicamentos para HAS ${ }^{9,10,12}$.

A alta prevalência de hipertensão arterial sistêmica autorreferida evidenciada neste estudo e os fatores associados presentes estão de acordo com estudos de âmbito nacional. Entre os fatores de risco estudados, destaca-se a presença de história familiar de hipertensão arterial sistêmica, excesso de peso e inatividade física. Assim, o estudo pode servir de subsídio para ações dos órgãos públicos e setores privados no enfrentamento efetivo desta realidade.

\section{REFERÊNCIAS}

1. World Health Organization. Envelhecimento ativo: uma política de saúde. Brasília: Organização Pan-Americana da Saúde; 2005.

2. World Health Organization. The World health report: 2004: changing history. Geneva: WHO; 2004.

3. Whelan J. Who calls for countries to shift from acute to chronic care. New British Med. 2002;324:1237. https://doi.org/10.1136/ bmj.324.7348.1237

4. Campos MO, Rodrigues NJF. Doenças crônicas não transmissíveis: Fatores de risco e repercussão na qualidade de vida. Rev Baiana Saúde Públ. 2009;33(4):561-81.

5. Sociedade Brasileira de Hipertensão. VI Diretrizes Brasileiras de Hipertensão. Arq Bras Cardiol. 2010;95(1 supl.1):1-51.

6. Bulsing FL, Oliveira KF, Rosa LMK, Fonseca L, Areosa SVC. A influência dos grupos de convivência sobre a auto-estima das mulheres idosas do município de Santa Cruz do Sul-RS. RBCEH. 2007;4(1):11-7.

7. Almeida EA, Madeira GD, Arantes PMM, Alencar MA. Comparação da qualidade de vida entre idosos que participam e idosos que não participam de grupos de convivência na cidade de Itabira-MG. Rev Bras Geriatr Gerontol. 2010;13(3):435-43. https://doi.org/10.1590/ S1809-98232010000300010

8. Gontijo ÉE. Qualidade de vida, apoio social e depressão em idosos: relação com habilidades sociais. Rev Saúde Pesq. 2015;1(1):2.

9. Associação Brasileira de Empresas de Pesquisa. Dados com base no Levantamento Sócio Econômico 2008 - IBOPE. São Paulo: ABEP; 2010.

10. Guidelines for Data Processing and Analysis of the IPAQ [Internet]. 2008. [capturado 2014 Ago 4]; Disponível em: www.ipaq.ki.se/ scoring/htm

11. Organização Pan-Americana da Saúde. XXXVI Reunión del Comité Asesor de Investigaciones em Salud - Encuestra Multicêntrica Salud Bienestar y Envejecimento (sabe) em América Latina y el Caribe - Informe preliminar. Washington (DC): WHO; 2002.

12. Andrade SSA, Stopa SR, Brito AS, Chueri PS, Szwarcwald CL, Malta DC. Prevalência de hipertensão arterial autorreferida na população brasileira: análise da Pesquisa Nacional de Saúde, 2013. Epidemiol Serv Saúde. 2015;24(2):297-304. https://doi.org/10.5123/S167949742015000200012

13. Malta DC, Stopa SR, Andrade SSCA, Szwarcwald CL, Silva Júnior JB, Reis AAC. Cuidado em saúde em adultos com hipertensão arterial autorreferida no Brasil segundo dados da Pesquisa Nacional de Saúde, 2013. Rev Bras Epidemiol. 2015;18(supl. 2):109-22. https:// doi.org/10.1590/1980-5497201500060010 
14. Chobanian AV, Bakris GL, Black HR, Cushman WC, Green LA, Izzo JL Jr, Jones DW, Materson BJ, Oparil S, Wright JT Jr, Roccella EJ; National Heart, Lung, and Blood Institute Joint National Committee on Prevention, Detection, Evaluation, and Treatment of High Blood Pressure; National High Blood Pressure Education Program Coordinating Committee. The Seventh Report of the Joint National Committee on Prevention, Detection, Evaluation, and Treatment of High Blood Pressure: the JNC 7 report. JAMA. 2003;289(19): 2560-72. https://doi.org/10.1001/jama.289.19.2560

15. Ferreira CCC, Peixoto MRG, Barbosa MA, Silveira EA. Prevalência de fatores de risco cardiovascular em idosos usuários do Sistema Único de Saúde de Goiânia. Arq Bras Cardiol. 2010;95(5):621-8. https:// doi.org/10.1590/S0066-782X2010005000141

16. Soar C. Prevalência de fatores de risco cardiovascular em idosos não institucionalizados. Rev Bras Geriatr Gerontol. 2015;18(2):385-95. https://doi.org/10.1590/1809-9823.2015.14072

17. Zattar C, Boing AF, Giehl MWC, d'Orsi E. Prevalência e fatores associados à pressão arterial elevada, seu conhecimento e tratamento em idosos no sul do Brasil. Cad Saúde Pública. 2013;29(3):507-21. https://doi.org/10.1590/S0102-311X2013000300009

18. GBD 2015 Risk Factors Collaborators. Global, regional, and national comparative risk assessment of 79 behavioural, environmental and occupational, and metabolic risks or clusters of risks, 1990-2015: a systematic analysis for the Global Burden of Disease Study 2015.
Lancet. 2016,388(8):1659-724. https://doi.org/10.1016/S01406736(16)31679-8

19. Barreto SM, Passos VMA, Firmo JOA, Guerra HL, Vidigal PG, LimaCosta MF. Hypertension and clustering of cardiovascular risk factors in a community in Southeast Brazil - The Bambuí Health and Ageing Study. Arq Bras Cardiol. 2001;77:576-81. https://doi.org/10.1590/ S0066-782X2001001200008

20. Freitas OC, Resende de Carvalho F, Marques Neves J, Veludo PK, Silva Parreira R, Marafiotti Gonçalves R, Arenales de Lima S, Bulgarelli Bestetti R. Prevalência de hipertensão na população urbana de Catanduva, no Estado de São Paulo, Brasil. Arq Bras Cardiol. 2001;77:9-21. https://doi.org/10.1590/S0066782X2001000700002

21. Barroso WKS, Jardim PCBV, Vitorino PV, Bittencourt A, Miquetichuc F. Influência da atividade física programada na pressão arterial de idosos hipertensos sob tratamento não-farmacológico. Rev Assoc Med Bras. 2008;54(4):328-33. https://doi.org/10.1590/S010442302008000400018

22. Malta DC, Silva Jr JB. O Plano de ações estratégicas para o enfrentamento das doenças crônicas não transmissíveis no Brasil e a definição das metas globais para o enfrentamento dessas doenças até 2025: uma revisão. Epidemiol Serv Saude. 2013;22(1):151-64. https://doi.org/10.5123/S1679-49742013000100016 